\title{
Compatibility of quantum states
}

\author{
David Poulin* and Robin Blume-Kohout ${ }^{\dagger}$ \\ Los Alamos National Laboratory, Theoretical Division, MS-B210, Los Alamos, New Mexico 87545
}

(Dated: October 26, 2018)

\begin{abstract}
We introduce a measure of the compatibility between quantum states - the likelihood that two density matrices describe the same object. Our measure is motivated by two elementary requirements, which lead to a natural definition. We list some properties of this measure, and discuss its relation to the problem of combining two observers' states of knowledge.
\end{abstract}

PACS numbers: 03.65.Ta, 02.50.-r, 03.67.-a

The quantum superposition principle induces a qualitative difference between classical and quantum states of knowledge. The state of a quantum system can be fully specified, yet not predict with certainty the outcome of a measurement - a state of affairs which has only the observers' ignorance as classical analogue. In quantum mechanics, incomplete knowledge is represented by a mixed density matrix, which corresponds imperfectly to a classical distribution; the "quantum uncertainty" of pure states combines with the "classical uncertainty" of a distribution to yield an object which can be represented by different decompositions or preparations.

The fidelity of two quantum states [1] $\rho_{A}$ and $\rho_{B}$,

$$
F\left(\rho_{A}, \rho_{B}\right)=\operatorname{Tr}\left\{\sqrt{\sqrt{\rho_{A}} \rho_{B} \sqrt{\rho_{A}}}\right\}
$$

(or more precisely $F^{2}$ ) measures the likelihood that various measurements made on the two states will obtain the same result. Thus, fidelity is a measure of similarity between states which does not distinguish between classical and quantum uncertainty.

In this letter, we introduce compatibility, a measure similar to fidelity, but which compares two observers' states of knowledge, not the results of the measurements which they could do. We want the compatibility to measure classical admixture, while treating different pure states as fundamentally different: if two observers claim to have complete knowledge of a system, their descriptions had better agree completely. Hence, a compatibility measure $C\left(\rho_{A}, \rho_{B}\right)$ should satisfy the two following requirements:

1. When $\left[\rho_{A}, \rho_{B}\right]=0$ (classical mixture) the compatibility should be equal to the fidelity.

2. The compatibility of incompatible states should be 0 .

While our first requirement should be transparent, the second sounds tautological, and requires further explanation. Consider two observers (Alice and Bob) whose respective states of knowledge are described by $\rho_{A}$ and $\rho_{B}$.

\footnotetext{
*Email address: poulinda@iro.umontreal.ca

${ }^{\dagger}$ Email address: rbk@socrates.Berkeley.edu
}

(Throughout this letter, we use subscript $k$ to designate either $A$ or B.) Brun, Finkelstein, and Mermin [2] defined Alice's and Bob's descriptions to be compatible if and only if they could be describing the same physical system. They then addressed the following question: under what conditions are $\rho_{A}$ and $\rho_{B}$ compatible? Their answer is quite simple: $\rho_{A}$ and $\rho_{B}$ are compatible if and only if the intersection of their supports, $\mathcal{S}=\mathcal{S}\left(\rho_{A}\right) \cap \mathcal{S}\left(\rho_{B}\right)$, is nonempty. The support $\mathcal{S}(\rho)$ of a density matrix $\rho$ is the complement of its null space $\mathcal{N}(\rho)$; to obtain the projector $P_{\mathcal{S}(\rho)}$ onto $\mathcal{S}(\rho)$, diagonalize $\rho$ and replace each nonzero eigenvalue with 1 . Thus, $\rho_{A}$ and $\rho_{B}$ are compatible if $P_{\mathcal{S}\left(\rho_{A}\right)} P_{\mathcal{S}\left(\rho_{B}\right)}$ has at least one unit eigenvalue. In other words, two states of knowledge are incompatible if between them they rule out all possible pure states.

With this definition, state $\rho_{A}=|0\rangle\langle 0|$ is compatible with both state $\rho_{B}=\epsilon|0\rangle\langle 0|+(1-\epsilon)| 1\rangle\langle 1|$ and state $\rho_{B}^{\prime}=$ $(1-\epsilon)|0\rangle\langle 0|+\epsilon| 1\rangle\langle 1|$ as long as $0<\epsilon<1$. Nevertheless as $\epsilon \rightarrow 0$, it is clear that the compatibility of $\rho_{A}$ and $\rho_{B}$ should vanish while that of $\rho_{A}$ and $\rho_{B}^{\prime}$ should approach unity. The definition of [2] makes no distinction between these two cases and this is what originally motivated the present work.

Now that requirement 2 has been clarified, we can proceed with the definition of the compatibility measure.

Definition 1 Let $\mathfrak{B}_{0}(\mathcal{H})$ be the set of all density matrices on Hilbert space $\mathcal{H}$. For $\rho \in \mathfrak{B}_{0}(\mathcal{H})$, define $\mathcal{P}(\rho)$ as the set of preparations of $\rho$ :

$$
\mathcal{P}(\rho)=\left\{P: \int_{\mathfrak{B}_{0}(\mathcal{H})} P(\sigma) \sigma d \sigma=\rho\right\}
$$

where the $P$ are probability distributions over $\mathfrak{B}_{0}(\mathcal{H})$. Then, the compatibility of $\rho_{A}$ and $\rho_{B} \in \mathfrak{B}_{0}(\mathcal{H})$ is defined as

$$
C\left(\rho_{A}, \rho_{B}\right)=\max _{\substack{P_{A} \in \mathcal{P}\left(\rho_{A}\right) \\ P_{B} \in \mathcal{P}\left(\rho_{B}\right)}} \int_{\mathfrak{B}_{0}(\mathcal{H})} \sqrt{P_{A}(\sigma) P_{B}(\sigma)} d \sigma,
$$

the integral representing the classical fidelity $F\left(P_{A}, P_{B}\right)$ (or statistical overlap) of two classical distributions $P_{A}$ and $P_{B}$.

Lemma 1 All distributions $P \in \mathcal{P}(\rho)$ must vanish outside $\mathfrak{B}_{0}(\rho)$ : the set of density matrices with support restricted to $\mathcal{S}(\rho)$ (this is a slightly abusive notation). 
Proof Let $P(\sigma)$ be a preparation of $\rho$. We can separate $\rho$ in two parts:

$$
\begin{aligned}
\rho & =\int_{\mathfrak{B}_{0}^{\perp}(\rho)} P_{k}(\sigma) \sigma d \sigma+\int_{\mathfrak{B}_{0}(\rho)} P_{k}(\sigma) \sigma d \sigma \\
& =p \rho^{\prime}+(1-p) \rho^{\prime \prime}
\end{aligned}
$$

where $\rho^{\prime}$, by definition, has support on $\mathcal{N}(\rho), \rho^{\prime \prime}$ has support strictly on $\mathcal{S}(\rho)$ and $p=\int_{\mathfrak{B}_{0}^{\perp}(\rho)} P_{k}(\sigma) d \sigma$. If $p \neq$ 0 , there exists $\psi \in \mathcal{N}(\rho)$ such that $\rho$ does not annihilate $\psi$. This contradicts the definition of $\mathcal{N}(\rho)$, so we conclude that $p=0$ and therefore $P$ is restricted to $\mathfrak{B}_{0}(\rho)$.

Theorem 1 Definition 1 satisfies both of our requirements.

\section{Proof}

1. If $\rho_{A}$ commutes with $\rho_{B}$, then they have orthogonal decompositions onto the same set of pure states: $\rho_{A}=$ $\sum_{i} a_{i}\left|\phi_{i}\right\rangle\left\langle\phi_{i}\left|; \rho_{B}=\sum_{i} b_{i}\right| \phi_{i}\right\rangle\left\langle\phi_{i}\right|$. Thus $C\left(\rho_{A}, \rho_{B}\right) \geq$ $\sum_{i} \sqrt{a_{i} b_{i}}=F\left(\rho_{A}, \rho_{B}\right)$. Later (see P4) we show that $C\left(\rho_{A}, \rho_{B}\right) \leq F\left(\rho_{A}, \rho_{B}\right)$; therefore for commuting density matrices $C\left(\rho_{A}, \rho_{B}\right)=F\left(\rho_{A}, \rho_{B}\right)$.

2. If $\rho_{A}$ and $\rho_{B}$ are incompatible, their supports are disjoint, which implies that $P_{A}(\sigma)$ and $P_{B}(\sigma)$ are restricted to disjoint sets-implying that $C\left(\rho_{A}, \rho_{B}\right)=0$.

Note that this measure is not the only on which satisfies our two requirements. For example, define

$$
D_{n}\left(\rho_{A}, \rho_{B}\right)=\operatorname{Tr}\left\{\left[\left(\rho_{A}\right)^{1 / 2 n}\left(\rho_{B}\right)^{1 / n}\left(\rho_{A}\right)^{1 / 2 n}\right]^{n}\right\} .
$$

Clearly, $D_{n}\left(\rho_{a}, \rho_{B}\right)=F\left(\rho_{A}, \rho_{B}\right)$ when $n=1$ or for any $n$ when $\left[\rho_{A}, \rho_{B}\right]=0$, so it satisfy our first requirement. For the second requirement, notice that

$$
\lim _{n \rightarrow \infty} D_{n}\left(\rho_{A}, \rho_{B}\right) \leq \lim _{n \rightarrow \infty} \operatorname{Tr}\left\{\left[P_{\mathcal{S}\left(\rho_{A}\right)} P_{\mathcal{S}\left(\rho_{B}\right)}\right]^{n}\right\}
$$

which is 0 if $P_{\mathcal{S}\left(\rho_{B}\right)} P_{\mathcal{S}\left(\rho_{B}\right)}$ has no unit eigenvalue, i.e. if $\rho_{A}$ and $\rho_{B}$ are incompatible. Therefore $D\left(\rho_{A}, \rho_{B}\right)=$ $\lim _{n \rightarrow \infty} D_{n}\left(\rho_{A}, \rho_{B}\right)$ is a valid measure of compatibility.

Definition 11 can also be generalized to

$$
E_{\alpha}\left(\rho_{A}, \rho_{B}\right)=\max _{\substack{P_{A} \in \mathcal{P}\left(\rho_{A}\right) \\ P_{B} \in \mathcal{P}\left(\rho_{B}\right)}} \int_{\mathfrak{B}_{0}(\mathcal{H})}\left[P_{A}(\sigma)\right]^{\alpha}\left[P_{B}(\sigma)\right]^{1-\alpha} d \sigma
$$

$0<\alpha<1$ which is the Rényi overlap of $P_{A}$ and $P_{B}$, the fidelity corresponding to the special case $\alpha=1 / 2$. This definition allows for an asymmetry between Alice and Bob which can be useful when one of the participant is more trustworthy than the other.

Although these alternative definitions offers some interesting features, we shall concentrate on Definition 1 in the following. (Superscript $D$ and $E$ indicates that the results also hold for measure $D\left(\rho_{A}, \rho_{B}\right)$ and $E_{\alpha}\left(\rho_{A}, \rho_{B}\right)$ respectively, the proofs are given for $C\left(\rho_{A}, \rho_{B}\right)$ only.)
Theorem $2^{E}$ To compute the compatibility of two states, it is sufficient to maximize over pure state preparations. In other words

$$
C\left(\rho_{A}, \rho_{B}\right)=\max _{\substack{Q_{A} \in \mathcal{Q}\left(\rho_{A}\right) \\ Q_{B} \in \mathcal{Q}\left(\rho_{B}\right)}} \int_{\mathfrak{B}_{0}^{1}(\mathcal{H})} \sqrt{Q_{A}(\psi) Q_{B}(\psi)} d \psi
$$

where $\mathfrak{B}_{0}^{1}(\mathcal{H})$ is the set of all pure states in $\mathcal{H}$ and $\mathcal{Q}(\rho)$ is the set of pure state preparations of $\rho$ :

$$
\mathcal{Q}(\rho)=\left\{Q: \int_{\mathfrak{B}_{0}^{1}(\mathcal{H})} Q(\psi)|\psi\rangle\langle\psi| d \psi=\rho\right\},
$$

$Q$ are probabilities distributions on $\mathfrak{B}_{0}^{1}(\mathcal{H})$.

Proof Choose a standard preparation for $\sigma \in \mathfrak{B}_{0}(\mathcal{H}), \sigma=$ $\int_{\mathfrak{B}_{0}^{1}(\mathcal{H})} f_{\sigma}(\psi)|\psi\rangle\langle\psi| d \psi$ (e.g., eigendecomposition). Then

$$
\begin{aligned}
& \int_{\mathfrak{B}_{0}(\mathcal{H})} \sqrt{P_{A}(\sigma) P_{B}(\sigma)} d \sigma \\
= & \int_{\mathfrak{B}_{0}(\mathcal{H})} \int_{\mathfrak{B}_{0}^{1}(\mathcal{H})} \sqrt{P_{A}(\sigma) f_{\sigma}(\psi) P_{B}(\sigma) f_{\sigma}(\psi)} d \sigma d \psi \\
\leq & \int_{\mathfrak{B}_{0}^{1}(\mathcal{H})} \sqrt{Q_{A}(\psi) Q_{B}(\psi)} d \psi
\end{aligned}
$$

since fidelity can only increase under the marginalization $Q_{k}(\psi)=\int_{\mathfrak{B}_{0}(\mathcal{H})} P_{k}(\sigma) f_{\sigma}(\psi) d \sigma$.

Theorem 3 When one of the two states is pure (say $\left.\rho_{B}\right), C\left(\rho_{A}, \rho_{B}\right)=\sqrt{p}$ where $p$ is given by

$$
p=\min _{q \in[0,1]}\left\{q: \operatorname{det}_{\mathcal{S}\left(\rho_{A}\right)}\left\{\rho_{A}-q \rho_{B}\right\}=0\right\}
$$

if $\rho_{B}$ lies within $\mathcal{S}\left(\rho_{A}\right)$ and $p=0$ otherwise.

Proof There is a unique preparation for $\rho_{B}: P_{B}(\sigma)=$ $\delta\left(\sigma-\rho_{B}\right)$. The maximum value of $q$ for which we can write $\rho_{A}=q \rho_{B}+(1-q) \sigma$ (with $\sigma$ a valid density matrix) is $p$. The result follows.

Theorem $4^{E}$ Any local maximum of $F\left(P_{A}, P_{B}\right)$ over $\mathcal{P}\left(\rho_{A}\right) \otimes \mathcal{P}\left(\rho_{B}\right)$ is a global maximum.

Proof Fidelity is a concave function: $F\left(\lambda P_{A}+\right.$ $\left.[1-\lambda] P_{A}^{\prime}, P_{B}\right) \geq \lambda F\left(P_{A}, P_{B}\right)+[1-\lambda] F\left(P_{A}^{\prime}, P_{B}\right)$. The sets $\mathcal{P}\left(\rho_{A}\right)$ and $\mathcal{P}\left(\rho_{B}\right)$ are convex: any convex combinations of valid probability distributions of mean $\rho$ is also a valid probability distribution with mean $\rho$. The result follows automatically.

We now give a list of properties of the compatibility measure.

$\mathbf{P} 1^{D} C\left(\rho_{A}, \rho_{B}\right)$ is symmetric.

$\mathbf{P 2}^{D E}$ Compatibility is invariant under unitary transformation: $C\left(U \rho_{A} U^{\dagger}, U \rho_{B} U^{\dagger}\right)=C\left(\rho_{A}, \rho_{B}\right)$.

$\mathbf{P 3}^{D E}$ For pure states $C\left(\psi_{A}, \psi_{B}\right)=1$ if and only if $\left|\left\langle\psi_{A} \mid \psi_{B}\right\rangle\right|^{2}=1$ and 0 otherwise. 
$\mathbf{P} 4^{D}$ (Upper bound) $C\left(\rho_{A}, \rho_{C}\right) \leq F\left(\rho_{A}, \rho_{B}\right)$.

$\mathbf{P 5}^{D E} F\left(\rho_{A}, \rho_{B}\right)=0 \Rightarrow C\left(\rho_{A}, \rho_{B}\right)=0$ and $F\left(\rho_{A}, \rho_{B}\right)=$ $1 \Leftrightarrow C\left(\rho_{A}, \rho_{B}\right)=1 \Leftrightarrow \rho_{A}=\rho_{B}$.

P6 (Lower bound) $C\left(\rho_{A}, \rho_{B}\right) \geq r \sqrt{\epsilon_{A} \epsilon_{B}}$ where $\epsilon_{k}$ is the greatest value of $q$ for which one can write $\rho_{k}=\frac{q}{r} P_{\mathcal{S}}+$ $(1-q) \sigma$ with $\sigma$ being a valid density matrix, see $($ eq 12 , and $r=\operatorname{Tr}\left\{P_{\mathcal{S}}\right\}$ is the dimension of $\mathcal{S}=\mathcal{S}\left(\rho_{A}\right) \cap \mathcal{S}\left(\rho_{B}\right)$. (For compatible states, $\epsilon_{k} \geq \lambda_{k}^{0}$, the smallest nonzero eigenvalue of $\rho_{k}$.)

$\mathbf{P} 7^{E}$ (Multiplicativity) $C\left(\rho_{A} \otimes \rho_{A}^{\prime}, \rho_{B} \otimes \rho_{B}^{\prime}\right) \geq$ $C\left(\rho_{A}, \rho_{B}\right) C\left(\rho_{A}^{\prime}, \rho_{B}^{\prime}\right)$.

Proofs

P1, P2, and P3 are straightforward from Definition 11.

P4: Assume that $Q_{k}(\psi)$ are the optimal distributions given by Theorem 2. We choose $\vec{x} \in \mathbb{R}^{(2 N-2)}$ (where $N$ is the dimension of $\mathcal{H}$ ) as a parameterization for $\mathfrak{B}_{0}^{1}(\mathcal{H})$ : $\psi=\psi(\vec{x})$, and construct the purifications

$$
\left|\Psi_{k}\right\rangle=\int \sqrt{Q_{k}(\psi(\vec{x}))}|\psi(\vec{x})\rangle \otimes|\vec{x}\rangle d \vec{x}
$$

where $\vec{x}$ is now treated as a quantum continuous variable $\left\langle\vec{x} \mid \vec{x}^{\prime}\right\rangle=\delta\left(\vec{x}-\vec{x}^{\prime}\right)$ (e.g. position of a particle in a $N$-dimensional box $)$. Then $C\left(\rho_{A}, \rho_{B}\right)=\left\langle\Psi_{A} \mid \Psi_{B}\right\rangle \leq$ $F\left(\rho_{A}, \rho_{B}\right)$ since the fidelity is the maximum of this quantity over all purifications.

This proof introduces an interesting distinction between fidelity and compatibility. Fidelity is the optimal inner product between all purifications of $\rho_{A}$ and $\rho_{B}$. On the other hand, compatibility involves purifications of a very special kind (eq.13). All that is needed to transform compatibility into fidelity is to replace (eq.13) by

$$
\begin{aligned}
& \left|\Psi_{A}\right\rangle=\int \sqrt{Q_{A}(\psi(\vec{x}))}|\psi(\vec{x})\rangle \otimes U_{A}|\vec{x}\rangle d \vec{x} \\
& \left|\Psi_{B}\right\rangle=\int \sqrt{Q_{B}(\psi(\vec{x}))}|\psi(\vec{x})\rangle \otimes U_{B}|\vec{x}\rangle d \vec{x}
\end{aligned}
$$

for arbitrary unitary operators $U_{A}$ and $U_{B}$.

P5 follows from $F\left(\rho_{A}, \rho_{B}\right)=1 \Leftrightarrow \rho_{A}=\rho_{B}$, requirement 1 , and $\mathrm{P} 4$.

P6: We can choose a distribution where $\rho_{k}$ has probability $r \epsilon_{k}$ at the point $\sigma=P_{\mathcal{S}} / r$.

P7: The product of the optimal distributions for $C\left(\rho_{A}, \rho_{B}\right)$ and $C\left(\rho_{A}^{\prime}, \rho_{B}^{\prime}\right)$ are valid distributions over the combined Hilbert space but might not be optimal. We do not know if this inequality can be reduced to an equality. In other words, it is possible that the optimal distribution for $\rho_{A} \otimes \rho_{A}^{\prime}$ and $\rho_{B} \otimes \rho_{B}^{\prime}$ involve non product states.

It is worth mentioning that no smooth function of the compatibility satisfying $f(C)=1 \Leftrightarrow C=0$ and $f(C)=0 \Leftrightarrow C=1$ can be used to build a metric on $\mathfrak{B}_{0}(\mathcal{H})$. This is best illustrated by the following 2dimensional example. Assume states $\rho_{+}$and $\rho_{-}$are pure, derived from $\left|\psi_{ \pm}\right\rangle=\cos \epsilon|0\rangle \pm \sin \epsilon|1\rangle$, and $\rho_{0}=$ $(1-\epsilon)|0\rangle\langle 0|+\epsilon| 1\rangle\langle 1|$ where $\epsilon \rightarrow 0$. One can easily verify that $C\left(\rho_{+}, \rho_{-}\right)=0$ and $C\left(\rho_{+}, \rho_{0}\right)=C\left(\rho_{-}, \rho_{0}\right)=1-O(\epsilon)$ so $f\left(C\left(\rho_{+}, \rho_{-}\right)\right)=1>f\left(C\left(\rho_{+}, \rho_{0}\right)\right)+f\left(C\left(\rho_{-}, \rho_{0}\right)\right) \rightarrow 0$ as $\epsilon \rightarrow 0$. This is in contrast with classical distributions: when $\left[\rho_{A}, \rho_{B}\right]=0, \cos ^{-1} F\left(\sqrt{\rho_{A} \rho_{B}}\right)$ is a valid distance measure $[3]$.

Measurement - Suppose that Alice and Bob acquire their knowledge of $\rho_{A}$ and $\rho_{B}$ through measurement. These states will always be compatible: incompatible knowledge acquired through measurement would indicate an inconsistency in quantum theory ${ }^{1}$. For example, they can each be given many copies of a quantum system in state $\rho$ of which they initially have no knowledge except the dimension. They carry out independent measurements on those copies and, with the help of Bayesian rules, update their description of the system (see 4 and references therein). As mentioned earlier, their descriptions will always be compatible. Nevertheless, a low compatibility could result as a consequence of one of the following situations: $i$ ) they were given copies of different states; ii) their measurement apparatus are miscalibrated; or iii) they are in a very improbable branch of the Universe.

These eventualities cannot be detected by the fidelity of $\rho_{A}$ and $\rho_{B}$. For example, suppose that, for a 2-level system,

$$
\begin{gathered}
\rho_{A}=(1-\epsilon)|0\rangle\langle 0|+\frac{\epsilon}{2} 11 \\
\rho_{B}=(1-\epsilon)|+\rangle\langle+|+\frac{\epsilon}{2} 11,
\end{gathered}
$$

where $|+\rangle=\frac{1}{\sqrt{2}}(|0\rangle+|1\rangle)$. As the observers' knowledge becomes more and more accurate $(\epsilon \rightarrow 0)$, the compatibility goes to 0 , indicating one of the three situations listed above. On the other hand, fidelity saturates at $F^{2}=1 / 2$, which is the same as if both Alice and Bob had a vague knowledge of the state, e.g.

$$
\begin{aligned}
& \rho_{A}=\left(\frac{1}{2}+a\right)|0\rangle\left\langle 0\left|+\left(\frac{1}{2}-a\right)\right| 1\right\rangle\langle 1| \\
& \rho_{B}=\left(\frac{1}{2}-a\right)|0\rangle\left\langle 0\left|+\left(\frac{1}{2}+a\right)\right| 1\right\rangle\langle 1|
\end{aligned}
$$

with $a=\sqrt{2} / 4$. This clearly illustrates the fact that fidelity makes no distinction between classical and quantum uncertainty.

Combining knowledge - Now, suppose Alice and Bob want to pool their information. If $C\left(\rho_{A}, \rho_{B}\right)=0$ (which cannot result from measurement), their "knowledge" is contradictory. When $C\left(\rho_{A}, \rho_{B}\right)>0$, however, they can combine their states of knowledge to get a new density matrix $\rho_{A B}$. This issue has recently been studied by Jacobs [5] but with the only conclusion that $\rho_{A B}$ should lie in $\mathcal{S}\left(\rho_{A}\right) \cap \mathcal{S}\left(\rho_{B}\right)$.

We propose that the state obtained from combining two states of knowledge should be the one which is maximally compatible with both of them. This requires a

\footnotetext{
${ }^{1}$ Incompatible knowledge could emerge as a consequence of the finite accuracy of the measurement apparatus: nevertheless, such limitations should be taken into account in the state estimation.
} 
definition of three-way compatibility:

$$
\begin{aligned}
& C\left(\rho_{A}, \rho_{B}, \rho_{C}\right)= \\
& \max _{\substack{P_{A} \in \mathcal{P}\left(\rho_{A}\right) \\
P_{B} \in \mathcal{P}\left(\rho_{B}\right) \\
P_{C} \in \mathcal{P}\left(\rho_{C}\right)}} \int_{\mathfrak{B}_{0}(\mathcal{H})} \sqrt[3]{P_{A}(\sigma) P_{B}(\sigma) P_{C}(\sigma)} d \sigma .
\end{aligned}
$$

Hence, our rule for combining states of knowledge reads

$$
\rho_{A B}=\operatorname{Argument}\left(\max _{\rho} C\left(\rho_{A}, \rho_{B}, \rho\right)\right) ;
$$

in the eventuality that the maximum over $\rho$ is not unique, one can discriminate with a maximum entropy $S(\rho)$ criteria which is well motivated in the current context. For any fixed $P_{A}$ and $P_{B}$, the $P_{C}$ that optimizes (eq.18) is proportional to the geometric average of $P_{A}$ and $P_{B}$. Therefore, defining $\tilde{P}_{A}$ and $\tilde{P}_{B}$ as the distributions which optimized equation (3), we get

$$
\rho_{A B}=\int_{\mathfrak{B}_{0}(\mathcal{H})} P_{A B}(\sigma) \sigma d \sigma
$$

where $P_{A B}=\sqrt{P_{A} P_{B}} / C\left(\rho_{A}, \rho_{B}\right)$. Furthermore, there is a simple relation between the optimal three-way compatibility and the compatibility of the two original descriptions: $C\left(\rho_{A}, \rho_{B}, \rho_{A B}\right)^{3}=C\left(\rho_{A}, \rho_{B}\right)^{2}$.

Knowledge-Knowledge of a quantum system can take many forms; as Bennett expresses it,

It is possible to know or possess a quantum state in infinitely many physically inequivalent ways, ranging from complete classical knowledge, through possession of a single specimen of the state, to weaker and less compactly embodiable forms such as the ability to simulate the outcome of a single POVM measurement on the state. [6]

The compatibility measurement of (eq.3) is meaningful when we consider classical description of the quantum states; the quantum fidelity (eq.11) corresponds to a situation where single specimens of the quantum states are available (respectively "knowledge of the quantum" and "quantum knowledge"). One can define compatibility measurements according to the type of knowledge one is dealing with. For example, we can define the compatibility between a state $\rho$ and an ensemble $\left\{q_{j}, \sigma_{j}\right\}$ as $\max _{\mathcal{P}(\rho)} F(P, Q)$ where $Q(\sigma)=\sum_{j} q_{j} \delta\left(\sigma-\sigma_{j}\right)$. While the pure state $|+\rangle=\frac{1}{\sqrt{2}}(|0\rangle+|1\rangle)$ is compatible with the ensemble $E_{1}=\{(1, p|0\rangle\langle 0|+(1-p)| 1\rangle\langle 1|)\}$, it is incompatible with the ensemble $E_{2}=\{(p,|0\rangle\langle 0|),((1-p),|1\rangle\langle 1|)\}$, even if they are preparations of the same state.

An ensemble embodies more knowledge than its associated (average) state. In our prescription for combining knowledge, we have assumed that all of Alice's and Bob's knowledge was encapsulated in their respective density matrices. Note that all knowledge can be represented in this form by including ancillary systems [e.g. eq.(13)].

Suppose, instead, that both Alice's and Bob's states of knowledge are represented by the ensemble $E_{1}$. Obviously, their combined density matrix should be $\rho_{A B 1}=$ $p|0\rangle\langle 0|+(1-p)| 1\rangle\langle 1|$. On the other hand, when both their states of knowledge are $E_{2}$, Bayesian rules would suggest that their combined state should be $\rho_{A B 2}=p^{2}|0\rangle\langle 0|+$ $(1-p)^{2}|1\rangle\langle 1|$ (with proper normalization) - but this assumes that their knowledge was acquired independently [5]. If their knowledge came from a redundant source, the Bayesian rule would then yield state $\rho_{A B 1}$, as would our prescription.

Hence, this illustrates that our rule for combining states of knowledge assumes no more information than what is encapsulated in the density matrices. Furthermore, it can quite simply be adapted to different forms of knowledge, either through the use of ancillary systems or of generalized compatibility measures.

Acknowledgements - The authors would like to acknowledge Howard Barnum, Kurt Jacobs, Harold Ollivier, and Wojciech Zurek for discussions on this subject. RBK would also like to thank Todd Brun for discussions and inspiration.
[1] R. Jozsa, J. of Mod. Opt. 41, 12 (1994) p.2315.

[2] T.A. Brun, J. Finkelstein, and N. D. Mermin, Phys, Rev. A 65, (2002) p.32315.

[3] C.A. Fuchs, Distinguishability and Accessible Information in Quantum Theory, PhD thesis, University of New Mexico, Albuquerque (1995).
[4] R. Schack, T.A. Brun, and C.M. Caves, Phys. Rev. A 64, (2001) p.14305.

[5] K. Jacobs, arXiv: quant-ph/0201096 (2002).

[6] C.H. Bennett, Fourth Workshop on Quantum Information Processing, Amsterdam, (2001). 